\title{
Spectrum of disease caused by Rhodococcus equi in human immunodeficiency virus infection: Report of a case and review of the literature
}

SAndra K Willsie-Ediger, DO, James F Stanford, MD, Gary A SALZMAN, MD, FCCP, DAVID M BAMBERGER, MD

ABSTRACT: Since the first report of Rhodococcus equi infection in an acquired immune deficiency syndrome patient in 1986, seven additional cases have been described. A patient is described in whom the diagnosis was delayed due to misidentification of the organism as an atypical mycobacterial species. The literature regarding $R$ equi infection in persons infected with the human immunodeficiency virus is reviewed. The most common presentation is one of a chronic, indolent pulmonary infiltrative disease $(78 \%)$. Fever $(78 \%)$, cough $(67 \%)$, and hemoptysis $(44 \%)$ are frequently present. Coexistent opportunistic illnesses are common
$(67 \%)$. In the laboratory identification of this organism. it is important to communicate the clinical setting to the microbiologist and to recognize the potential for the organism to be overlooked as normal flora or a contaminant, or misidentified as an organism with similar phenotypic characteristics (Nocardia species or a rapidly growing mycobacterium). Based on experience in foals, therapy with erythromycin and rifampin is suggested. Can J Infect Dis 1990;1(3): 101-107

Key Words: AIDS, HIV infection, Rhodococcus equi
$\mathrm{R}$ HODOCOCCUS EQUI INFECTION IN PATIENTS WITH human immunodeficiency virus (HIV) infection was first reported in 1986 (1). Since that time, there have been several reports of $R$ equi infection in HIV infected patients (2-8), with illness not necessarily localized or limited to the respiratory

Departments of Pulmonary Diseases and Infectious Diseases, University of Missouri-Kansas City. Kansas City. Missouri, USA

Correspondence and reprints: Sandra K Willsie-Ediger. DO. School of Medicine. University of Missouri-Kansas City. 2411 Holmes, Kansas City. Missouri 64108. USA

Received for publication March 8, 1990. Accepted June 11. 1990 tract. The diagnosis of $R$ equi illness in HIV infected patients is difficult and frequently delayed. In addition, misidentification of this organism as a rapidly growing Mycobacterium or Nocardia species has occurred $(5,9)$. Treatment of $R$ equi infections in HIV infected patients is similarly challenging. A case is reported in which diagnosis and appropriate treatment of $R$ equi infection in an acquired immune deficiency syndrome (AIDS) patient was delayed due to lack of clinician familiarity with the organism and misidentification as a rapidly growing mycobacterium. In addition, a review of the literature regarding the presentation, diagnosis and treatment of this op- 
portunistic infection in HIV infected patients is presented.

\section{CASE PRESENTATION}

A 44-year-old homosexual male AIDS patient was admitted to Truman Medical Center-West with a six week history of night sweats, nonproductive cough and increasing dyspnea. A chest roentgenogram revealed a right lower lobe infiltrate with cavitation (Figure 1). Bronchoscopy was performed and transbronchial biopsies of the right lower lobe obtained. Bacterial cultures revealed 'normal flora' and significant growth $(2+)$ of Escherichia coli. Transbronchial biopsies revealed acute and chronic inflammation only; special stains/cultures for acid-fast bacilli, fungal organisms, and Pneumocystis carinii were negative. Blood cultures were considered to be contaminated when they were reported to contain 'Corynebacterium species, not $J K$ ' in one of six bottles. Therapy for presumed bacterial lung abscess was instituted with ampicillin and metronidazole with clinical response and stabilization of the chest roentgenogram. The patient was discharged on oral therapy with these agents.

Readmission two weeks later was prompted by recurrence of fever and dyspnea and the development of an intractable cough. Results of the chest roentgenogram were unchanged. Bronchoscopy was repeated at that time. Quantitative protected brush catheter culture results revealed significant growth of Corynebacterium and Staphylococcus species, coagulase negative. Acid-fast bacilli smears were positive on bronchoalveolar lavage fluid and bronchial washings. Antituberculous therapy in the form of isoniazid $300 \mathrm{mg}$ daily, rifampin $600 \mathrm{mg}$ daily, ethambutol $900 \mathrm{mg}$ daily and pyridoxine $50 \mathrm{mg}$ daily was instituted. Clinically, the patient responded to medical therapy; fever resolved and cough lessened.

The acid-fast bacilli culture was reported as positive three weeks later due to an apparent atypical mycobacterial species and the culture was sent to the state reference laboratory for identification and sensitivity. The patient was discharged in an improved state.

Five weeks later, notification was received that the organism was a rapidly growing mycobacterium which could not be speciated. Outpatient evaluation of the patient at that time revealed him to be clinically stable in reference to respiratory symptoms; he continued to have rare episodic fevers in association with weight loss. Biochemical evaluation revealed an alkaline phosphatase of $1230 \mathrm{iu} / \mathrm{L}$, and the patient was readmitted for evaluation of possible isoniazid hepatitis versus infiltrative liver disease and for consideration of

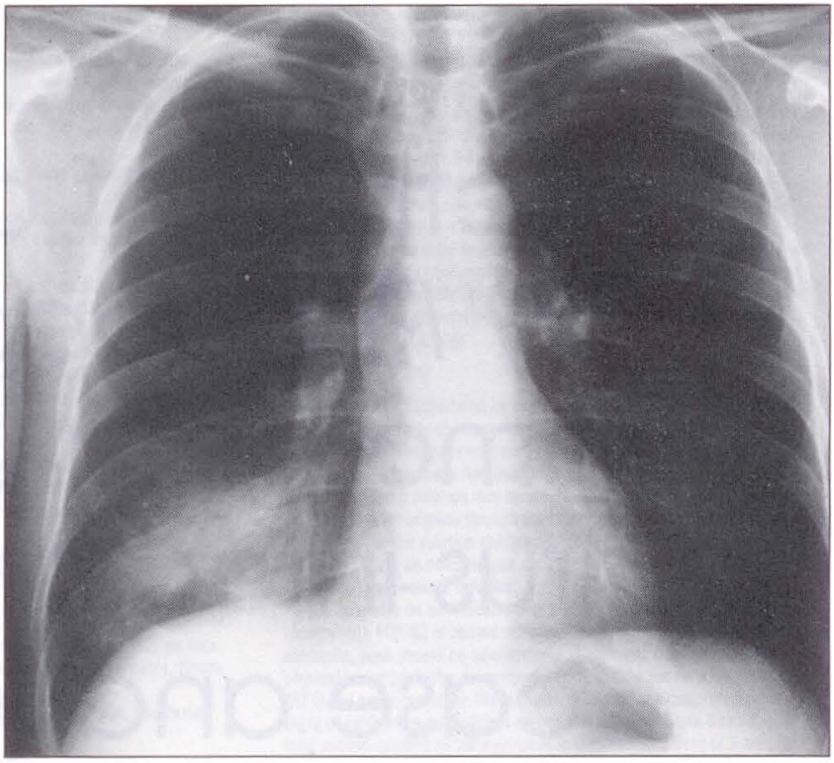

Figure 1) Posteroanterior roentgenogram revealing right lower lobe infiltrate with cavitation

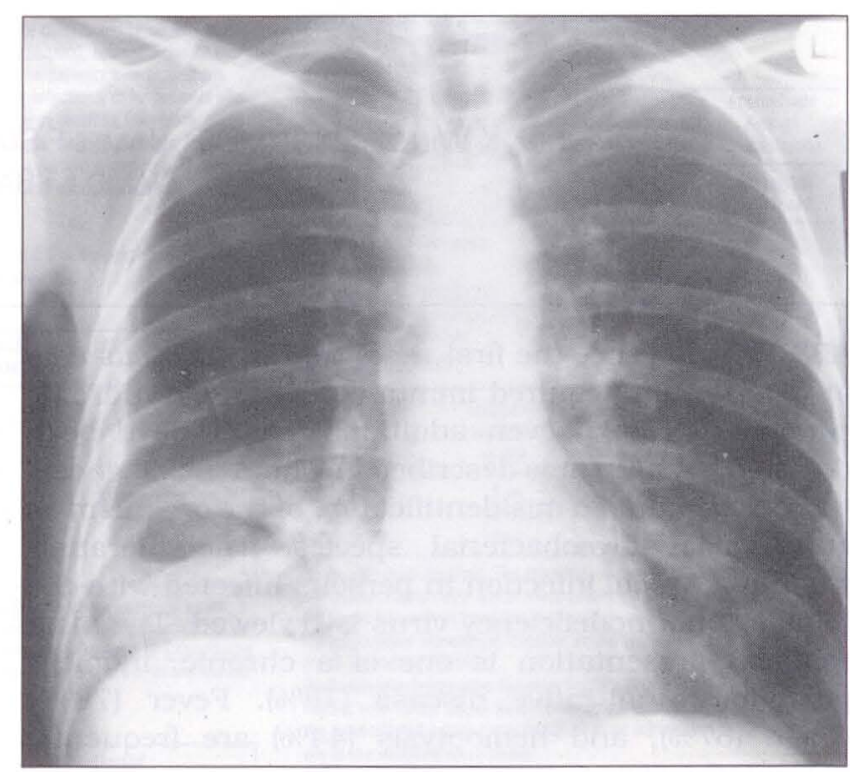

Figure 2) Posteroanterior roentgenogram revealing persistence/worsening of right lower lobe infiltration with associated pleural effusion

alternative therapy. Antituberculous therapy was discontinued. Chest roentgenographic examination revealed a persistent right lower lobe cavity and a new right pleural effusion (Figure 2). Thoracentesis was performed which revealed pleural fluid pH 8.0, lactate dehydrogenase 722 $\mathrm{iu} / \mathrm{L}$, protein $3.1 \mathrm{~g}$ and glucose $61 \mathrm{mg} / \mathrm{dL}$. Acidfast bacilli smears and cultures were negative. A liver biopsy was performed with findings of mild steatosis and minimal focal fibrosis; normal architecture was retained. Special stains for microorganisms were negative. Clinically, the 


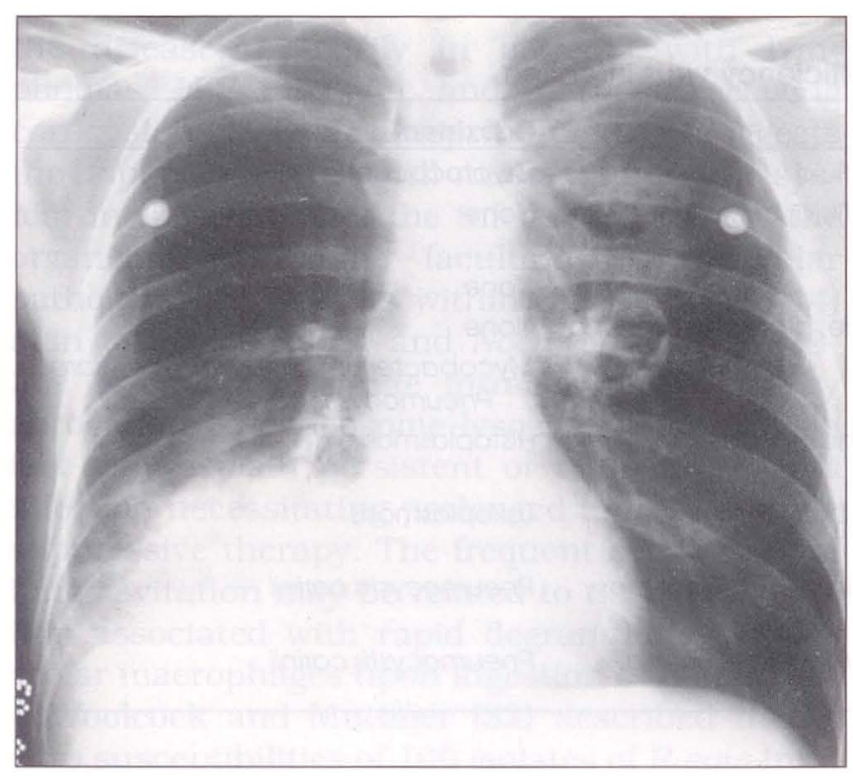

Figure 3) Posteroanterior roentgenogram revealing worsening of right lower lobe process and new left upper lobe infiltrate/cavitation

patient remained stable off antituberculous therapy and, at his request, was discharged without further therapy.

Pleural fluid and blood culture results were subsequently reported (at five days) to be growing a 'diphtheroid' organism in pure culture, and the patient was recontacted. At this time, the diagnosis of $R$ equi infection was entertained. The organism found in pleural fluid and blood and the organism previously reported to be an atypical mycobacterial species were sent to the Center for Disease Control (CDC - Atlanta, Georgia) for definitive identification. Pending identification and sensitivity of the organism, the patient was treated with intravenous vancomycin $1 \mathrm{~g}$ every 12 $\mathrm{h}$, and gentamicin $100 \mathrm{mg}$ every $8 \mathrm{~h}$ for 14 days. The patient requested to be discharged, refusing long term parenteral antibiotic therapy. He was subsequently discharged on oral erythromycin, $500 \mathrm{mg}$ every $6 \mathrm{~h}$. Definitive identification of the diphtheroid organism as $R$ equi was made six weeks later.

Maintained on long term erythromycin, monthly aerosolized pentamidine, and zidovudine therapy, the patient improved and stabilized. He was readmitted, however, five months later with increasing symptoms of dyspnea and cough. Chest roentgenographic findings revealed worsening of the right lower lobe process and development of a new left upper lobe cavity and infiltrate (Figure 3). Parenteral antibiotic therapy was reinstituted with vancomycin and gentamicin. Quantitative bacterial cultures of the protected brush catheter obtained at bronchoscopy revealed significant growth of Pseudomonas aeruginosa, Klebsiella pneumoniae and viridans streptococci. No rhodococcus or 'diphtheroids' were noted on Gram stain or culture. Bronchoalveolar lavage fluid was, however, acid-fast bacilli smear positive; due to contamination, the acid-fast bacillus was not identifiable as to genus and species. Blood cultures were negative. Despite the above therapy, the patient developed progressive respiratory failure and died five days later. Permission for post mortem examination was denied.

\section{DISCUSSION}

$R$ equi, a Gram-positive pleomorphic rod, formerly known as Corynebacterium equi, has been known to cause significant disease in animals for over 50 years (10-14). The first case of human disease caused by this organism was not reported until 1967 (15). Subsequent reports in humans have described disease primarily in immunocompromised hosts $(7,16-23)$, though two instances of $R$ equi-related infection in nonimmunocompromised hosts have been reported (2425).

$R$ equi-related infection in association with HIV infection was first reported in 1986, with seven additional cases described subsequently. A review of these cases in addition to the case described herein (nine patients), allows for characterization of the spectrum of disease caused by $R$ equi in patients with HIV infection.

$R$ equi-related infection was the presenting feature (alone or in combination with other manifestations) of HIV infection/AIDS in five of the nine HIV infected individuals (cases 1-4 and 6). Weingarten et al (2), because of the rarity of $R$ equi infection in normal hosts, has suggested that $R$ equi infection be added to the list of AIDS-defining conditions in HIV infected individuals.

Pulmonary disease was a prominent feature in seven of the nine patients (Table 1). In cases 5 and $6, R$ equi was implicated as causing gastroenteritis, lymphadenitis, soft tissue infection and sepsis in the presence of reportedly normal chest roentgenograms. Bloodstream infections were documented in all but one of the patients (the report of case 4 does not mention whether or not blood cultures were obtained). Fever occurred in $78 \%$, cough in $67 \%$, hemoptysis in $44 \%$ and chest pain in $44 \%$. Patients with strictly extrapulmonary disease presented with complaints of bloody diarrhea, back pain and paralumbar mass. Coexistent diseases were present in $67 \%$, including cryptococcosis, syphilis, $P$ carinii pneumonia, disseminated Mycobacterium avium-intracellulare, and toxoplasmosis.

Chest roentgenographic features of $R$ equi- 
TABLE 1

Spectrum of Rhodococcus equi disease in human immunodeficiency virus infection

\begin{tabular}{|c|c|c|c|c|}
\hline Reference & Case & Sites of disease & Laboratory recovery & Coexistent diseases \\
\hline 1 & 1 & Lung, pleura, bloodstream & Blood, sputa, pleural fluid & Cryptococcosis, syphilis \\
\hline \multirow[t]{2}{*}{2} & 2 & Lung, pleura, bloodstream & $\begin{array}{l}\text { Blood, sputa, pleural fluid, bronchial } \\
\text { washing }\end{array}$ & None \\
\hline & 3 & Lung, pleura, bloodstream & Blood, sputa & None \\
\hline 3 & 4 & Lung & Sputa, alveolar lavage, bronchial washing & None \\
\hline \multirow[t]{2}{*}{4} & 5 & $\begin{array}{l}\text { Gastroenteritis, lymph- } \\
\text { adenitis, bloodstream }\end{array}$ & Blood, stool & $\begin{array}{l}\text { Mycobacterium avium-intracellulare, } \\
\text { Pneumocystis carinii }\end{array}$ \\
\hline & 6 & $\begin{array}{l}\text { Lymphadenitis, soft tissue, } \\
\text { bloodstream }\end{array}$ & Blood, lymph node, mass aspirate & Histoplasmosis \\
\hline 5 & 7 & Lung, pleura, bloodstream & $\begin{array}{l}\text { Blood, sputa, pleural fluid, bronchial } \\
\text { washing }\end{array}$ & Toxoplasmosis \\
\hline $6,7,8$ & 8 & Lung, bloodstream & $\begin{array}{l}\text { Blood, sputa, bronchial washing, autopsy } \\
\text { specimen }\end{array}$ & Pneumocystis carinii \\
\hline $\begin{array}{l}\text { Present } \\
\text { study }\end{array}$ & 9 & Lung, pleura, bloodstream & $\begin{array}{l}\text { Blood, pleural fluid, bronchial washing, } \\
\text { alveolar lavage }\end{array}$ & Pneumocystis carinii \\
\hline
\end{tabular}

related infection in HIV infected patients are variable. Reported findings have included cavitary lesions (five patients), infiltrates (four patients), pleural effusion (four patients), pleural thickening (two patients), normal roentgenogram (two patients), and pseudotumour (one patient). In reference to lobar predilection in patients presenting with pulmonary involvement, the left upper lobe was involved in $57 \%$, and the right lower lobe in $43 \%$.

Bronchoscopy is frequently performed in HIV infected patients in order to diagnose pulmonary processes accurately. Bronchoscopic abnormalities were specifically mentioned in two cases of $R$ equi infection in HIV infected patients (cases 4 and 7). In both reports, bronchial orifices were partially or completely obstructed $(3,5)$.

Since biopsies were not uniformly performed or reported in HIV infected patients with $R$ equi illness, it is impossible to predict with certainty what the predominant histopathological features are. Existing reports, however, have cited acute necrotizing pneumonitis (2), inflammatory pseudotumour composed primarily of histiocytes (3), and mixed acute and chronic inflammation consistent with organizing pneumonia (2). Similar to the histological appearance of $M$ avium-intracellulare in patients with AIDS, macrophages intracellularly packed with $R$ equi organisms have also been described in HIV infected patients $(1,4)$.

The subacute/chronic presentation of $R$ equirelated infection in HIV infected patients (cough, fever and sweats coexisting with pulmonary infiltrates which may cavitate or become associated with empyema) is very similar to the presentations described in non-HIV infected immunocompromised patients. Compared to non-HIV infected patients, HIV infected patients with $R$ equi-related infection are more frequently bacteremic (eight of eight [100\%] HIV infected patient versus five of 14 [36\%] non-HIV infected patients) and have hemoptysis more frequently in association with pulmonary involvement (four of seven [57\%] HIV infected patients versus one of 14 [7\%] non-HIV infected patients). A history of farm animal exposure may be less frequently elicited from HIV infected patients with $R$ equi infection $(25 \%)-$ exposure history was not given in five of the nine HIV infected patients - as opposed to non-HIV infected persons $(69 \%)(7,19,21,23)$.

Though microbiological characteristics and identification methods for $R$ equi have been developed and described $(8,18-19,24,26-28)$, difficulty in diagnosing this illness persists. This may result from a lack of clinician familiarity with both organism and disease presentation, lack of distinction by the microbiology laboratory of this organism from 'normal flora,' or misidentification of the organism (19,21-23). Cavitary lung disease observed on chest roentgenogram may also mimic the presentation of tuberculosis. In HIV infected patients with $R$ equi infection, the acid-fast stain was positive in five of six patients where this stain was used and reported (cases 1,3,5,8 and 9). Patient 5 also had coincident $M$ avium-intracellulare infection. A positive acid-fast bacilli smear and growth of the organism on Lowenstein-Jensen media prompted use of antituberculous agents in patient 9 and in at least two other cases $(9,23)$. The organism has been confused with nonpathogenic Corynebacterium species when isolated from blood or sputum (24,26-28) and has also been misidentified as nocardia (case 7 ).

Histological evidence that $R$ equi is found intracellularly within macrophages in patients with $(1,4)$ and without AIDS (19), and the occurrence of 
the disease, primarily in patients with lymphomas, HIV infection, and in those receiving corticosteroids or azathioprine $(19,21)$, suggests the importance of the cell-mediated immune system in the control of the infection. In foals, the organism acts as a facultative intracellular pathogen that persists within macrophages (14) akin to mycobacteria and Nocardia species (2931). $R$ equi may evade intracellular killing by abrogation of phagosome-lysosome fusion. This fact may explain persistent or relapsing $R$ equi infection necessitating prolonged and/or chronic suppressive therapy. The frequent occurrence of lung cavitation may be related to tissue destruction associated with rapid degranulation by alveolar macrophages upon ingestion of $R$ equi.

Woolcock and Mutimer (32) described the in vitro susceptibilities of 100 isolates of $R$ equi from animal sources in 1980 (32). The most active agents in vitro were penicillin G, doxycycline, erythromycin, lincomycin and the aminoglycosides. In contrast with the susceptibilities reported from animal isolates, $R$ equi isolates from humans have been uniformly sensitive to vancomycin, aminoglycosides and erythromycin, variably sensitive to chloramphenicol, clindamycin and sulfonamides, and almost always resistant to penicillin, cephalothin and semisynthetic penicillins. Rifampin susceptibility (minimal inhibitory concentration $0.19 \mu \mathrm{g} / \mathrm{mL}$ ) was reported by Samies (1). In several reports, the organism was initially susceptible to penicillins and developed resistance during therapy (1$8,19,21,23)$. Bactericidal activity of an antimicrobial regimen directed against this organism (vancomycin - peak 1:32, trough 1:16) has been documented in only one case in humans (1).

Synergy studies have been limited to equine isolates where rifampin combined with either erythromycin or penicillin resulted in synergy $(23,33,34)$. The combination of erythromycin and rifampin has been used extensively in equine infection since 1981 with a resultant decline in mortality from 50 to $100 \%$ (seen in the late 1970 s to early 1980s) to 0 to $12 \%$ (34-36). Hillerdal (21) reported the successful use of erythromycin in combination with rifampin in a patient with sarcoidosis and pulmonary/osseous $R$ equi infection.

The response to therapy in HIV infected patients with $R$ equi infection is difficult to interpret, as therapy has lacked uniformity, and several patients died but did not have post mortem examinations. In addition, drug dosages and duration of therapy have not always been reported. The overall response to therapy has been poor, and relapse is common. In four of the five patients who died in whom follow-up was reported (cases 3, 5, 8 and 9), $R$ equi infection may have been directly responsible or may have contributed to the patient's death. It is likely that even with 'optimal' treatment, the organism may only be inhibited rather than eradicated in HIV infected patients, evidenced by persistence of the organism and pulmonary disease despite therapy (cases $1,3,5$ and $8-9)$. The reported response to therapy in 15 non-HIV infected, immunocompromised patients appears more favourable; 12 of 15 (80\%) were cured of their infection $(7,19,21,23)$. (Survival $\mathrm{P}=0.023$ by Fisher's exact test in comparison to two of eight HIV infected patients.) In these patients, eight of nine (89\%) who received some form of surgical/drainage procedure (two alone, seven with antibiotic therapy), and four of six $(67 \%)$ treated with antibiotic therapy alone, survived $(7,19,21,23)$. Survival (alive at the end of reported follow-up ranging from six to 10 months) among HIV infected patients with $R$ equi infection who did not obviously die of other causes was $20 \%$ (one in five) in patients treated with antibiotics alone and 50\% (one in two) in those treated with surgery combined with antibiotics. The effects of surgical excision of localized infection, surgical or bronchoscopic drainage of parenchymal abscesses and needle/tube drainage of infection involving the pleural space on the outcome of $R$ equi infection are also difficult to interpret, as antibiotic regimens lacked uniformity, the numbers of patients receiving various procedures were small, and the procedures were not uniform in approach or time of application. Additionally, in some cases (eg, cases 1 and 4), the surgery was performed early for diagnostic rather than therapeutic purposes.

Recommendations for therapy from the literature have included consideration of surgical intervention (1) - particularly in isolated pulmonary or soft tissue disease - as well as antibiotic therapy with two parenteral agents (1-2) followed by a prolonged course of two oral agents to which the organism is sensitive (2). Complete resolution of disease in successfully treated non-HIV infected immunocompromised patients usually requires two to six months of therapy, and Van Etta et al (19) recommend continuing therapy at least until the organism can no longer be cultured and the patient is clinically stable. Based on the authors experience with the patient reported herein, the poor outcomes of patients previously reported, the experience in foals and in vitro studies, it is suggested that HIV infected patients with $R$ equi infection be treated with erythromycin and rifampin for several months, and that orally administered chronic suppressive therapy with these two 
agents be considered. The use of other regimens in patients failing an erythromycin/rifampin combination therapy should be guided by susceptibility and possibly other in vitro adjunctive testing. Surgical intervention should be considered and used for refractory cavitary disease or life threatening hemoptysis; drainage procedures should be employed when infection involves the pleural or other protected space. Clearly, further in vitro, animal model and controlled clinical studies are needed.

\section{CONCLUSIONS}

$R$ equi is a significant pathogen in HIV infected patients. Of the nine cases reported in the literature to date, seven have had pulmonary involvement and two strictly extrapulmonary disease. Clinical and roentgenographic features of $R$ equi disease in HIV infected patients, sites of involvement, coexistent diseases and laboratory identification of the organism have been summarized. Recognition of this organism's potential to cause significant, serious infection in the HIV infected population - and of the clinical features of this infection - may enable prompt diagnosis and early institution of therapy - factors which may improve an otherwise poor prognosis. When an HIV infected patient presents with a pulmonary infiltrate

\section{REFERENCES}

1. Samies JH, Hathaway BN, Echols RM, Veazey JM, Pilon VA. Lung abscess due to Corynebacterium equi. Report of the first case in a patient with the acquired immunodeficiency syndrome.

Am J Med 1986;80:685-8.

2. Weingarten JS, Huang DY, Jackman JD. Rhodo coccus equi pneumonia. An unusual early manifestation of the acquired immunodeficiency syndrome. Chest 1988;94:195-6.

3. Bishopric GA, Françoise d'Agay M, Schlemmer B, Sarfati E, Brocheriou C. Pulmonary pseudotumor due to Corynebacterium equi in a patient with the acquired immunodeficiency syndrome. Thorax 1988;43:486-7.

4. Fierer J, Wolf P, Seed L, Gay T, Noonan K, Haghighi P. Nonpulmonary Rhodococcus equi infections in patients with the acquired immunodeficiency syndrome. J Clin Pathol 1987;40:556-8.

5. Sonnet J, Wauters G, Zech F, Gigi J. Opportunistic Rhodococcus equi infection in an African AIDS case (1976-1981). Acta Clin Belg 1987;42:215-6.

6. Sane DC, Durack DT. Infection with Rhodococcus equi in AIDS. N Engl J Med 1986:314:56-7. (Lett)

7. MacGregor JR, Samuelson WM, Sane DC, Godwin JD. Opportunistic lung infection caused by Rhodococcus (Corynebacterium) equi. Radiology 1986;160:83-4.

8. Clover D, Parks B, Harrell LJ. Laboratory identification of Rhodococcus equi from a patient with AIDS. Lab Med 1989;20:491-3.

9. Rubin RH. Pneumonia in the compromised host. with or without cavitation, pleural disease, or extrapulmonary disease which remains unexplained, involvement by $R$ equi should be considered. Alternatively, when an unidentified Corynebacterium species or 'diphtheroid', a Nocardia species, or a rapidly growing acid-fast bacillus is recovered from an HIV infected patient, $R$ equi should again be strongly considered and empiric therapy instituted pending definitive identification. Bacteriology laboratories should begin identifying Gram-positive rods as to genus and species in specimens (eg, blood cultures, bronchoscopically obtained specimens, abscess aspirates and other specimens obtained from normally sterile sites) obtained from known HIV infected patients. Optimal therapy for $R$ equi infection is not known, and controlled clinical trials, animal model and in vitro studies are needed. Until further studies are available to guide treatment, it is recommended that combination therapy with erythromycin and rifampin be given over several months initially; long term oral suppressive therapy with these two agents should also be strongly considered. Surgical intervention and/or drainage procedures should be used in life threatening hemoptysis, localized soft tissue abscess and thoracic empyema, and should be considered for refractory pulmonary abscesses/cavitary disease.

In: Fishman AP, ed. Update: Pulmonary Diseases and Disorders. New York: McGraw-Hill, 1982:1-25.

10. Magnusson H. Pyaemia in foals caused by Corynebacterium equi. Vet Rec 1938;50:1459-68.

11. Oxenford CI, Ratcliffe RC, Ramsay GC. Rhodo coccus equi infections in a cat. Aust Vet J 1987;64:121.

12. Hillidge CJ. Review of Corynebacterium (Rhodo coccus) equi lung abscesses in foals: Pathogenesis, diagnosis and treatment. Vet Rec 1986;119:261-4.

13. Takai S, Fujimori T, Katsuzaki K, Tsubaki S. Ecology of Rhodococcus equi in horses and their environment on horsebreeding farms. Vet Microbiol 1987; 14:233-9.

14. Yager JA. The pathogenesis of Rhodococcus equi pneumonia in foals. Vet Microbiol 1987; 14:225-32.

15. Golub B, Falk G, Spink WW. Lung abscess due to Corynebacterium equi. Report of first human infection. Ann Intern Med 1967;66:1174-7.

16. Carpenter JL, Blom J. Corynebacterium equi pneumonia in a patient with Hodgkin's Disease. Am Rev Respir Dis 1976;114:235-9.

17. Gardner SE, Pearson T, Hughes WT. Pneumonitis due to Corynebacterium equi. Chest 1976;70:92-4.

18. Marsh JC, Von Graevenitz A. Recurrent Corynebac terium equi infection with lymphoma. Cancer 1973;114:147-9.

19. Van Etta LL, Filice GA, Ferguson RM, Gerding DN. Corynebacterium equi: A review of 12 cases of human infection. Rev Infect Dis 1983;5:1012-8.

20. Berg R, Chmel H, Mayo J, Armstrong D. Corynebacterium equi infection complicating neoplastic disease. Am J Clin Pathol 
1977:68:73-7.

21. Hillerdal G, Riesenfeldt-Orn I, Pedersen A, Ivanicova E. Infection with Rhodococcus equi in a patient with sarcoidosis treated with corticosteroids. Scand J Infect Dis 1988;20:673-7.

22. Savdie E, Pigott P, Jennis F. Lung abscess due to Corynebacterium equi in a renal transplant recipient. Med J Aust 1977;1:817-9.

23. LeBar WD, Pensler MI. Pleural effusion due to Rhodococcus equi. J Infect Dis 1986;154:919-20. (Lett)

24. Muller F, Schaal KP, Von Graevenitz A, et al. Characterization of Rhodococcus equi-like bacterium isolated from a wound infection in a noncompromised host. J Clin Microbiol 1988;26:618-20.

25. Thomsen VF, Henriques U, Magnusson M. Corynebacterium equi isolated from a tuberculoid lesion in a child with adenitis colli. Dan Med Bull 1968; 15:135-8.

26. Lipsky BA, Goldberger AC, Tompkins LS, Plorde JJ. Infections caused by nondiphtheria corynebacteria. Rev Infect Dis 1982;4:1220-35.

27. Coyle MB, Hollis DG, Growman NB. Corynebacterium species and other coryneform organisms. In: Lennette EH, Balows A, Hausler WJ Jr, Shadomy H.J, eds. Manual of Clinical Microbiology, 4th edn. Washington: American Society for Microbiology, 1985:199.

28. Goodfellow M. Genus Rhodococcus Zopf 1891, 28 AL. In: Sneath PHA, Mair NS, Sharope ME, Holt JG, eds. Bergey's Manual of Systemic Bacteriology,
Vol 2. Baltimore: Williams \& Wilkins, 1986:1472-81.

29. Armstrong JA, Hart PD. Response of cultured macrophages to Mycobacterium tuberculosis, with observations on fusion of lysosomes with phagosomes. J Exp Med 1971;134:713-40.

30. Davis-Scibienski C, Beamon BL. The interaction of Nocardia asteroides with rabbit alveolar macrophages: The association of virulence, variability, ultrastructural damage and phagosome lysosome fusion. Infect Immun 1980;28:610-9.

31. Wilson JP, Turner HR, Kirchmer KA, Chapman SW. Nocardial infections in renal transplant recipients. Medicine 1989;68:38-53.

32. Woolcock JB, Mutimer MD. Corynebacterium equi: In vitro susceptibility to twenty-six antimicrobial agents. Antimicrob Agents Chemother 1980;18:976-7.

33. Prescott JF, Nicholson VM. The effects of combinations of selected antibiotics on the growth of Corynebacterium equi. J Vet Pharmacol Ther 1984;7:61-4.

34. Hillidge CJ. Use of erythromycin-rifampin combination in treatment of Rhodococcus equi pneumonia. Vet Microbiol 1987; 14:337-42.

35. Prescott JF, Sweeney CR. Treatment of Coryne bacterium equi pneumonia in foals: A review. J Am Vet Med Assoc 1985; 187:725-7.

36. Sweeney CR, Sweeney RW, Divert J. Rhodococcus equi pneumonia in 48 foals: Response to antimicrobial therapy. Vet Microbiol 1987;14:329-36. 


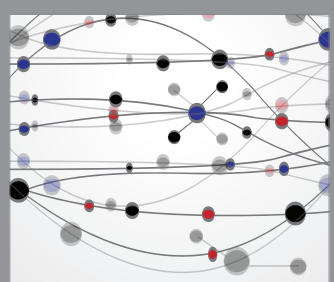

The Scientific World Journal
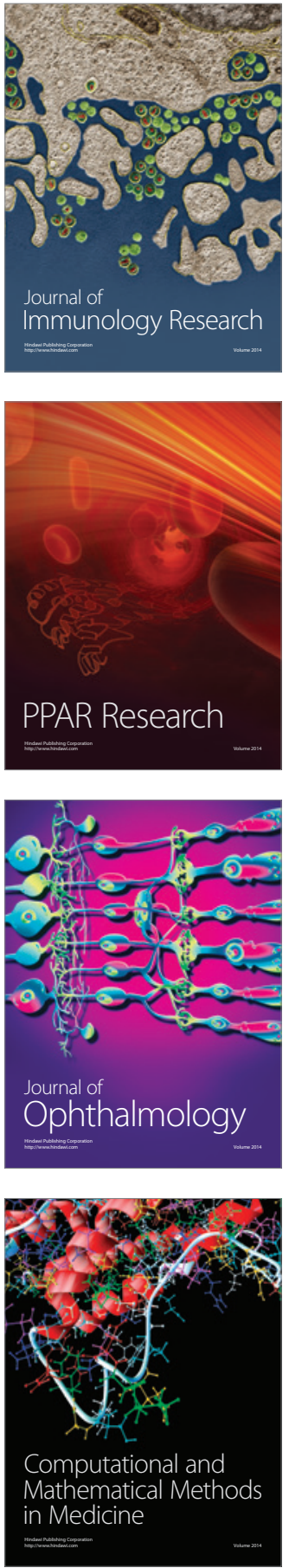

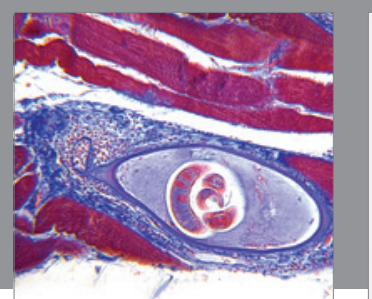

Gastroenterology Research and Practice

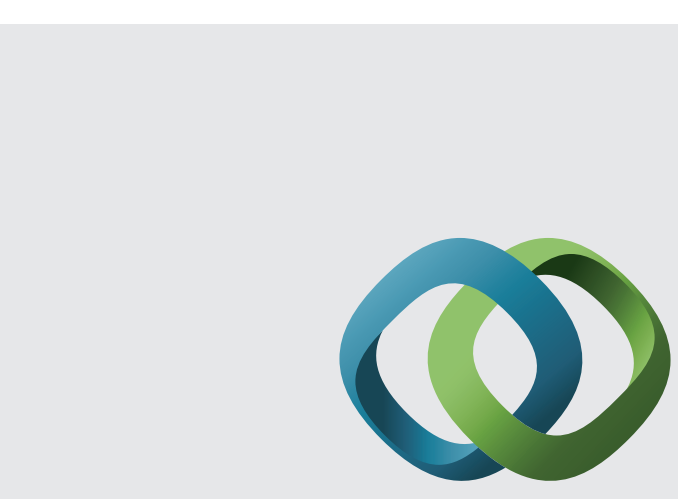

\section{Hindawi}

Submit your manuscripts at

http://www.hindawi.com
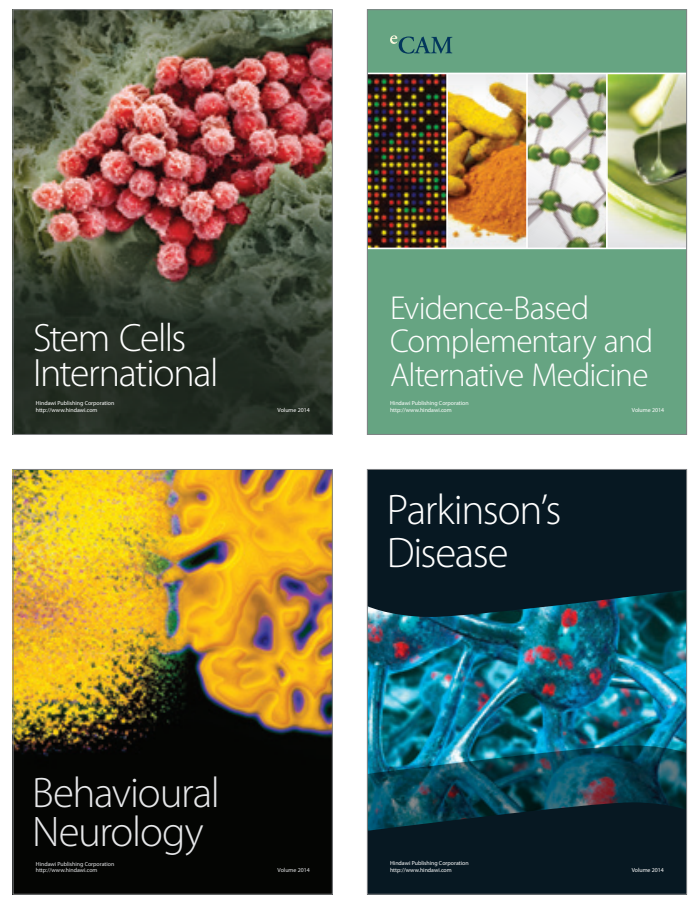
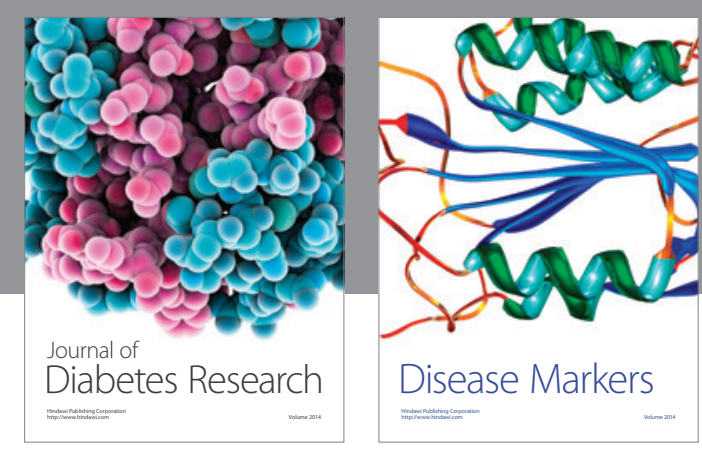

Disease Markers
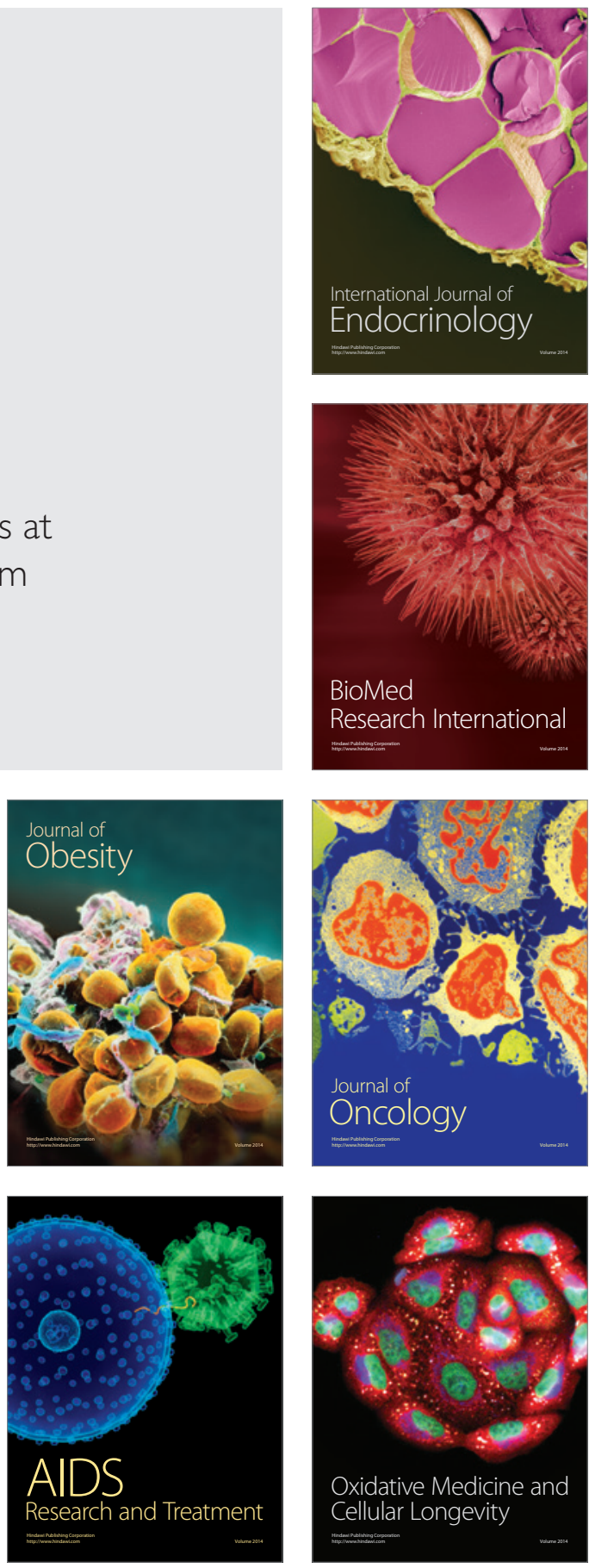